\title{
Modeling and Simulation of Torpedo Leading Angle Variety With Observation Errors
}

\author{
ZHAN Kai ${ }^{1, a}$, WANG Jing ${ }^{1, b}$ \\ ${ }^{1}$ Academy of Marine corps, Guangzhou 510430 Guangdong, China \\ azhankai5000@163.com, ${ }^{\mathrm{b}}$ cow423@126.com
}

Keywords: leading angle, straight-shooting, observation error

Abstract. The straight-shooting is the torpedo's important shooting method and its leading angle is key factor. Based on the theory of the torpedo straight-shooting triangle, modeling and discussing the torpedo leading angle variety caused by the target observation errors in straight-shooting. And simulations under different instances are presented to show the leading angle Variety.

\section{Introduction}

Torpedo is the important $r$ weapon in sea war and the most important weapon of the submarine. According to the homing method the torpedo can be classified such as the straight-running torpedo, the homing torpedo and the wired-guided torpedo. Even though the homing torpedo and the wired-guided torpedo are the main development direction of the submarine weapons, the straight-running torpedo's tactical significance can not be ignored. In some special conditions the straight-running torpedo or the straight-shooting method of the homing torpedo and the wired-guided torpedo are the more effective means on attacking targets.

In straight-shooting of the torpedo the leading angle is an important factor that influences the torpedo hit effect. In fact the target observation errors are the key factors that influence the straight-shooting torpedo's leading angle. In the paper in consideration of the target observation errors the model of the torpedo straight-shooting is establish and the effect of leading angle produced by the target observation errors are analyzed and simulated. The conclusion can provide reference on not only the straight-running torpedo's shooting but also the homing torpedo and the wired-guided torpedo's straight-running.

\section{The Model of the Torpedo's Straight-shooting}

For the sake of the main course and the key factors, the process of the torpedo straight-shooting is predigested. We assume that the target and the torpedo both has a currently movement without changing their speed and direction. The assument can be characterized the torpedo straight-shooting's process in despite of some inessential factor such as velocity of flow and so on. In Fig.1 shows the relatively situation of the target and the submarine when the torpedo is fired out of the submarine torpedo tube, M is the target's position and W is the torpedo's position when the torpedo is fired out of the submarine torpedo tube. In order to hit the target, torpedo must adopt an appropriate angle towards the target's motion direction. After a certain time, the target and the torpedo reach the point $\mathrm{C}$ at the same moment which $\mathrm{d}$ enotes the torpedo hit the target. $\triangle W C M$ is called the torpedo shooting triangle, $\mathrm{V}_{M}$ and $\mathrm{V}_{\mathrm{T}}$ are the speed of target and torpedo, $S_{M}$ and $S_{T}$ are the the target's and the torpedo's running distance, $\mathrm{D}_{S}$ is the distance between the target and the torpedo when the torpedo is fired out of the tube as shown in Fig. 1 .The angle $\varphi$ is called the torpedo leading angle, the angle $\lambda$ is the target shipboard angle and the angle $\theta$ is the torpedo hit angle. According to the torpedo shooting triangle and the law of sines, the torpedo's straight-shooting's motion equations are as follows, 


$$
\frac{S_{T}}{V_{T}}=\frac{S_{M}}{V_{M}}
$$

(1)

(2)

$$
\frac{S_{M}}{\sin \varphi}=\frac{S_{T}}{\sin \lambda}
$$

$$
\sin \varphi=\frac{S_{M}}{S_{T}} \cdot \sin \lambda=\frac{V_{M}}{V_{T}} \cdot \sin \lambda=m \cdot \sin \lambda
$$

(3)

Where $m$ is the velocity ratio of the target and the torpedo.

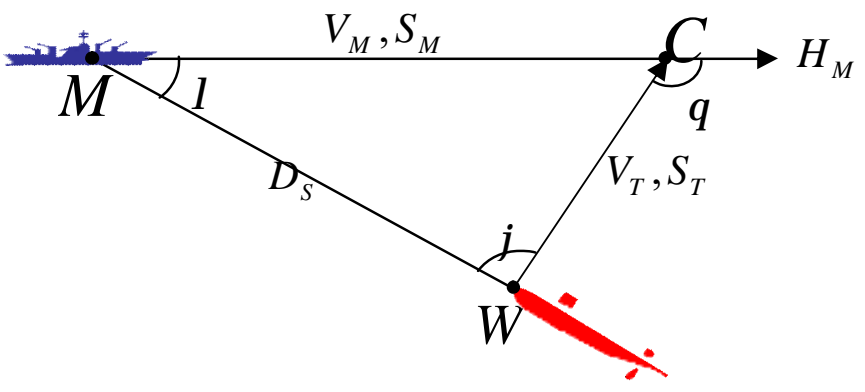

Fig.1 The Torpedo Shooting Triangle

\section{The Modle of the Torpedo Leading Angle Variety with the Target Observation Errors}

In the torpedo straight-shooting's process, the torpedo leading angle must be decided exactly according to the target motion elements such as the target's bearing and speed. The target motion elements are obtained by the submarine's observation. In actually the target motion elements obtained by observation have errors inescapability and bring some influence to the torpedo leading angle. As shown in Eq.3 the target's bearing and speed are the main factors that influence the torpedo leading angle. So we must analyse the errors of the target's bearing and speed and find out their influence to the torpedo leading angle. $\Delta \mathrm{V}_{\mathrm{M}}$ and $\Delta \lambda$ are the errors caused by the observation of the target's speed and the target's bearing, and $\Delta \varphi_{V}$ and $\Delta \varphi_{B}$ are the errors caused by the observation of the target's speed and the target's bearing.

\section{1 the Torpedo Leading Angle with the Target's Speed Error}

When observe the target's speed, the error $\Delta \mathrm{V}_{\mathrm{M}}$ ineluctability exits. In this section we analyse the torpedo leading angle caused only by the target's speed error. According to the geometrical analysis of the torpedo shooting triangle and the Eq.3, we can educe the relation equations as follows.

$$
\sin \left(\varphi \pm \Delta \varphi_{V}\right)=\frac{V_{M} \pm \Delta V_{M}}{V_{T}} \cdot \sin \lambda .
$$

Let $\Delta m=\Delta V_{M} / V_{T}$, Eq.4 can be translated as follows,

$$
\sin \left(\varphi \pm \Delta \varphi_{V}\right)=(m \pm \Delta m) \cdot \sin \lambda \text {. }
$$

According to the law of sines and Eq.5,we can educe that

$$
\sin \varphi \cdot \cos \Delta \varphi_{V} \pm \cos \varphi \cdot \sin \Delta \varphi_{V}=(m \pm \Delta m) \cdot \sin \lambda .
$$

When value of the error $\Delta \varphi_{V}$ is little comparatively, and $\cos \Delta \varphi_{V} \approx 1$.

$\mathrm{Q} \cos \Delta \varphi_{V} \approx 1$ and $\sin \varphi=m \cdot \sin \lambda$, 
$\therefore \sin \varphi \pm \cos \varphi \cdot \sin \Delta \varphi_{V}=(m+\Delta m) \cdot \sin \lambda$.

$\therefore \sin \Delta \varphi_{V} \cdot \cos \varphi=\Delta m \cdot \sin \lambda$,

$\therefore \sin \Delta \varphi_{V}=\frac{\sin \lambda}{\cos \varphi} \cdot \Delta m=\frac{\sin \lambda}{\cos \varphi} \cdot \frac{\Delta \mathrm{V}_{\mathrm{M}}}{V_{T}}$.

$\therefore \Delta \varphi_{V}=\arcsin \left(\frac{\sin \lambda}{\cos \varphi} \cdot \frac{\Delta \mathrm{V}_{\mathrm{M}}}{V_{T}}\right)$.

Eq.9 shows that the torpedo leading angle's change $\Delta \varphi_{V}$ caused only by the target's speed error.

\section{2 the Torpedo Leading Angle with the Target's Bearing Error}

When observe the target's bearing, the error $\Delta \lambda$ ineluctability exits also. In this section we analyse the torpedo leading angle caused only by the target's bearing error. According to the geometrical analysis of the torpedo shooting triangle and the Eq.3, we can also educe the relation equations as follows.

$$
\sin \left(\varphi \pm \Delta \varphi_{B}\right)=\mathrm{m} \cdot \sin (\lambda \pm \Delta \lambda) .
$$

According to the Eq.10 and the law of sines, Eq.10 can be translated as follows, $\sin \varphi \cdot \cos \Delta \varphi_{B} \pm \cos \varphi \cdot \sin \Delta \varphi_{B}=m \cdot(\sin \lambda \cdot \cos \Delta \lambda \pm \cos \lambda \cdot \sin \Delta \lambda)$.

When value of the error $\Delta \varphi_{B}$ and $\Delta \lambda$ are little comparatively, and $\cos \Delta \varphi_{B} \approx 1, \cos \Delta \lambda \approx 1$.

$\mathrm{Q} \cos \Delta \varphi_{B} \approx 1$ and $\cos \Delta \lambda \approx 1$

$\therefore \sin \varphi \pm \cos \varphi \cdot \sin \Delta \varphi_{B}=m \cdot(\sin \lambda \pm \cos \lambda \cdot \sin \Delta \lambda)$.

$\mathrm{Q} \sin \varphi=m \cdot \sin \lambda$

$\therefore \sin \Delta \varphi_{B}=m \cdot \frac{\cos \lambda}{\cos \varphi} \cdot \sin \Delta \lambda$.

$\therefore \Delta \varphi_{B}=\arcsin \left(m \cdot \frac{\cos \lambda}{\cos \varphi} \cdot \sin \Delta \lambda\right)$.

Eq.14 shows that the torpedo leading angle's change $\Delta \varphi_{B}$ caused only by the target's speed bearing.

\section{Simulation}

According to the Sec. 2 we assume four representative cases to simulate the torpedo leading angle errors caused by the target observation errors to find out some disciplinarians. Case 1 and Case 2 revel the torpedo leading angle variety mainly caused by the target's speed errors with different target speed and torpedo speed in Fig.2; Case 3 and Case 4 revel the torpedo leading angle variety mainly caused by the target's bearing errors with different target speed and torpedo speed in Fig.3.
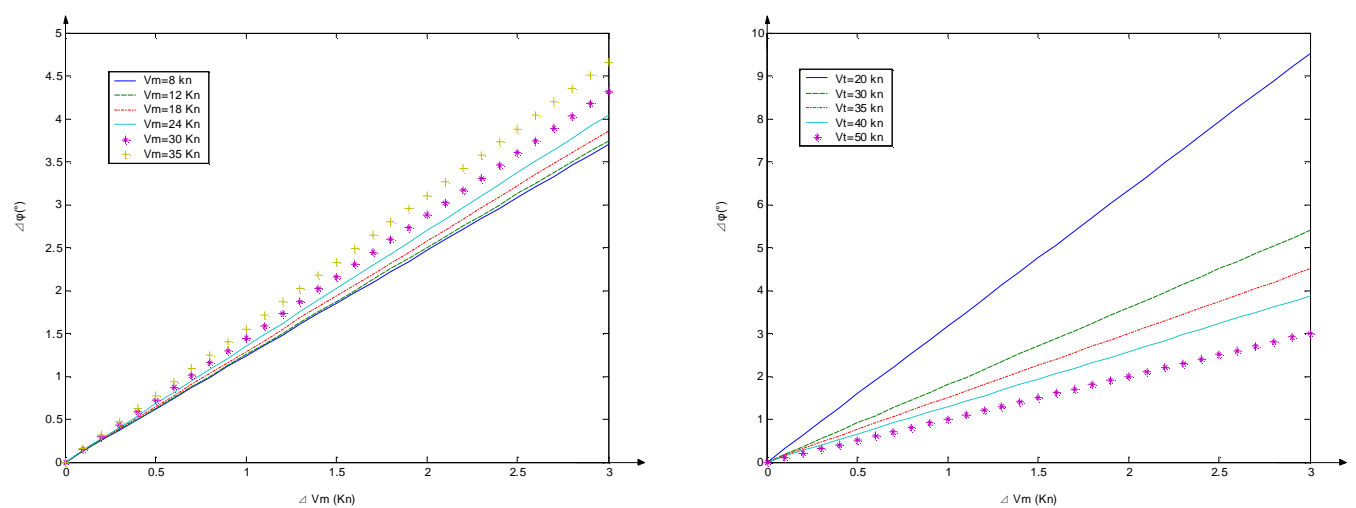

Fig.2 the Torpedo Leading Angle Errors mainly caused by the Target's Speed Errors 

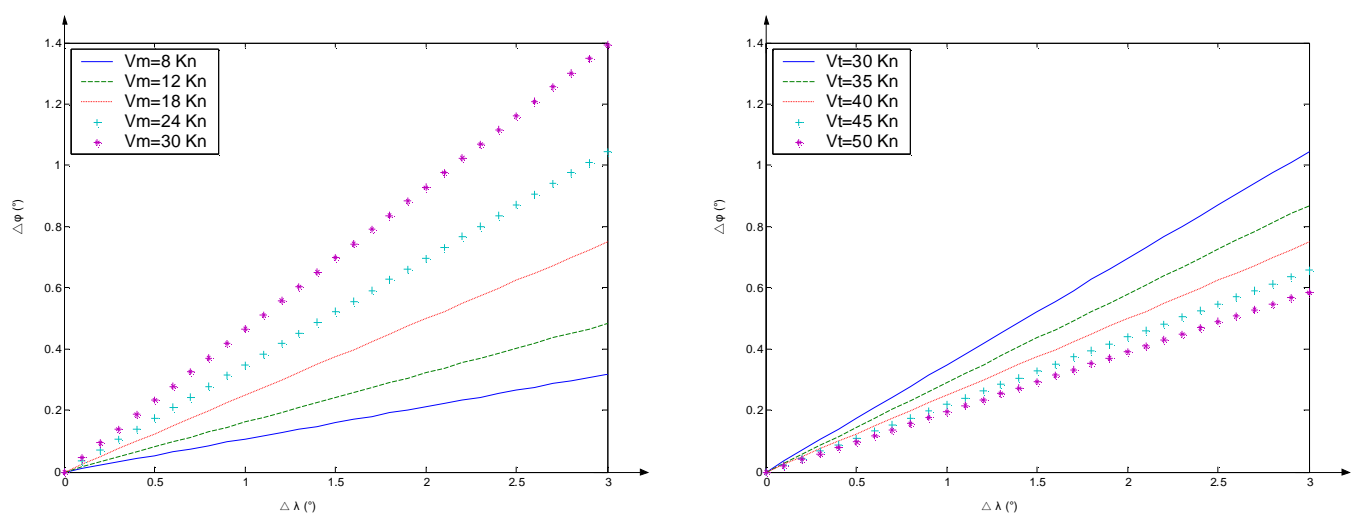

Fig.3 the Torpedo Leading Angle Errors mainly caused by the Target's Bearing Errors

\section{Conclusions}

The modeling and simulation of torpedo leading angle error in straight-shooting with the target observation errors such as speed and bearing are given in this paper. Through four representative instances of the torpedo leading error are discussed. The torpedo leading angle variety caused by the target's speed errors and the target's bearing errors show that leading angle variety increases with the target's speed and decreases with the torpedo speed. Therefore increasing the torpedo's speed can decrease the leading angle variety. Further work will focus on the hit probability with the target observation errors.

\section{References}

[1] TENG Zhao-xin: Calculation model and simulation of warship damage probability[J]. Journal of ChiHa Ordnance,2008.

[2] ZHANG Xu: Dispersion eharaeteristics of self-propelled mine position sequence coordinate[J].Fire and Control,2004.(in Chinese)

[3] ZHOU Cai-rong: Probabilitytistics theory and mathematicalsta [M]. (1984). (in Chinese)

[4] ZHANG Yu-wen: Torpedo overall design principles and methods[M].(1998)(in Chinese)

[5] JIA Yue: Mathematical model and simulation of torpedo weapon system effectiveness evaluation[J].( 2006). (in Chinese) 